OPEN ACCESS

Edited by:

Gianluca Serafini,

University of Genoa, Italy

Reviewed by:

Xenia Gonda,

Semmelweis University, Hungary

Priya Sharma,

University of Western Ontario,

Canada

Mirko Manchia,

Dalhousie University, Canada

*Correspondence:

Emmanuelle C. S. Bostock ebostock@utas.edu.au

Specialty section: This article was submitted to Mood and Anxiety Disorders, a section of the journal

Frontiers in Psychiatry

Received: 25 November 2016 Accepted: 10 July 2017

Published: 09 August 2017

Citation:

Bostock ECS, Kirkby KC, Garry MI and Taylor BVM (2017) Systematic

Review of Cognitive Function

in Euthymic Bipolar Disorder and Pre-Surgical Temporal Lobe Epilepsy.

Front. Psychiatry 8:133.

doi: 10.3389/fpsyt.2017.00133

\section{Systematic Review of Cognitive Function in Euthymic Bipolar Disorder and Pre-Surgical Temporal Lobe Epilepsy}

\author{
Emmanuelle C. S. Bostock ${ }^{1 *}$, Kenneth C. Kirkby ${ }^{2}$, Michael I. Garry ${ }^{1}$ and Bruce V. M. Taylor ${ }^{3}$ \\ ${ }^{1}$ Psychology, School of Medicine, University of Tasmania, Hobart, TAS, Australia, ${ }^{2}$ Psychiatry, School of Medicine, University \\ of Tasmania, Hobart, TAS, Australia, ${ }^{3}$ Menzies Institute for Medical Research, University of Tasmania, Hobart, TAS, Australia
}

Background: Bipolar disorder (BD) and temporal lobe epilepsy (TLE) overlap in domains including epidemiology, treatment response, shared neurotransmitter involvement and temporal lobe pathology. Comparison of cognitive function in both disorders may indicate temporal lobe mediated processes relevant to BD. This systematic review examines neuropsychological test profiles in euthymic bipolar disorder type I (BD-I) and pre-surgical TLE and compares experimental designs used.

Methods: A search of PubMed, PsychINFO, and Scopus using Preferred Reporting Items for Systematic Reviews and Meta-Analyses guidelines was conducted. Inclusion criteria were comparison group or pre- to post-surgical patients; reported neuropsychological tests; participants aged 18-60 years. Fifty six studies met criteria: 27 BD-I; 29 TLE.

Results: Deficits in BD-I compared to healthy controls $(\mathrm{HC})$ were in executive function, attention span and verbal memory. Deficits in TLE compared to HC were in executive function and memory. In the pre- to post-surgical comparisons, verbal memory in left temporal lobe ( $L T L)$ and, less consistently, visuospatial memory in right temporal lobe (RTL) epilepsy declined following surgery. BD-I studies used comprehensive test batteries in well-defined euthymic patients compared to matched HC groups. TLE studies used convenience samples pre- to post-surgery, comparing LTL and RTL subgroups, few included comparisons to HC (5 studies). TLE studies typically examined a narrow range of known temporal lobe-mediated neuropsychological functions, particularly verbal and visuospatial memory.

Conclusion: Both disorders exhibit deficits in executive function and verbal memory suggestive of both frontal and temporal lobe involvement. However, deficits in TLE are measured pre- to post-surgery and not controlled at baseline pre-surgery. Further research involving a head-to-head comparison of the two disorders on a broad range of neuropsychological tests is needed to clarify the nature and extent of cognitive deficits and potential overlaps.

Keywords: bipolar disorder, temporal lobe, focal seizures neuropsychology, cognition, epilepsy, systematic review 


\section{INTRODUCTION}

Bipolar disorder type I (BD-I) is typically characterized by episodes of mania and depression with inter-episode euthymia. A number of impairments have been noted in the euthymic phase of the illness including social, occupational functioning, and cognitive deficits $(1,2)$.

Most studies have examined cognitive deficits in euthymic patients with BD-I compared to healthy controls (HC). Five meta-analyses have reported impairments in cognitive domains of executive functioning (3-7), verbal memory $(3,5-7)$, visuospatial memory $(7)$, and attention $(4,6)$. One meta-analysis found no impairment of verbal memory and executive function in BD-I compared to $\mathrm{HC}$ (8). An individual patient data meta-analysis by Bourne et al. (9) reported impaired verbal memory and attention in euthymic BD-I patients relative to HC (9). The absence of an association between cognitive impairment and medication dose in euthymic BD-I patients suggest the effects of medication do not fully account for the cognitive impairments observed (4). These meta-analyses support the assumption that cognitive impairments exhibited in the euthymic phase are trait markers of the disorder.

An alternate research design is to compare and contrast cognitive deficits in BD-I to a reference condition which shares common features. For example, a meta-analysis comparing BD-I and schizophrenia reported more pronounced cognitive deficits in schizophrenia on measures of verbal fluency, verbal memory, executive function, visuospatial memory, mental speed, IQ, and concept formation (10). Similarly a single study comparing BD-I, obsessive compulsive disorder reported impaired verbal and episodic memory compared to HC (11). The BD-I group had greater impairments in learning word lists and delayed recall. These results suggest the importance of the temporal lobes in both disorders in the consolidation and retrieval of memories.

A single study compared BD-I with complex partial seizure disorder (12). It is noted that the classification of epilepsy syndromes has been subject to a number of iterations. In this review, we use the most commonly reported and widely understood term "temporal lobe epilepsy (TLE)" to incorporate the terms complex partial seizure disorder and focal seizures arising from the temporal lobes. The Jones et al. study reported greater impairment of executive function, attention and delayed verbal recall in the TLE group. These results should be interpreted with caution given the small and unequal sample sizes (BD-I $n=26$, TLE $n=9$ ). A case can be made for further exploration of similarities and differences between the neuropsychological test profiles seen in euthymic BD-I and interictal TLE. This is particularly so given the localizing pathology of TLE, which allows inferences to be made regarding the contribution of temporal lobe processes to the range of cognitive deficits reported in BD-I.

There are a number of established similarities between BD-I and TLE. These typically include a chronic course punctuated by episodic manifestations of mania and seizures, respectively. Other similarities include: the proposed involvement of kindling mechanisms (13); changes in neurotransmitters (excitatory amino acids, GABA, dopamine and serotonin), voltage-opened ion channels (sodium, calcium and potassium) and second messenger systems
(G-proteins, phosphatidylinositol, protein kinase C, myristoylated alanine-rich $\mathrm{C}$ kinase substrate), and treatment response to antiepileptic medications in both disorders (14).

In addition, episodes in both disorders commonly feature sensory, perceptual, cognitive, and affective changes (15) including depression (16). Epidemiological studies have shown that the proportion of BD-I among people with epilepsy is more than twice as high as in the general population (17) and that mania is more common in patients with TLE than in the general population (18). BD-I is also associated with comorbid epilepsy but not parental epilepsy (19). Episodes of mania in BD-I and seizures in TLE share precipitating factors including stress, sleep reduction and antidepressant medications (20).

The temporal lobes have also been the subject of neuroimaging research in both disorders. In BD-I many studies have investigated correlates of the disorder to specific brain regions. Meta-analyses of magnetic resonance imaging (MRI) studies have reported that BD-I is associated with right lateral ventricular enlargement (21) and an enlarged left amygdala (22). However, studies of temporal lobe size are inconsistent; with reported increases (23), reductions (24) or no differences (25-28) likely reflecting the difficulties of defining and measuring the volume of individual cerebral lobes on MRI.

In TLE, MRI studies have reported structural brain abnormalities in the hippocampus, entorhinal cortex (29), thalamus (30), and fornix (31). On voxel-based morphometry, TLE is associated with gray matter pathology in the hippocampus, cingulum, thalamus, and frontal lobes. White matter reductions ipsilateral to the seizure focus were also found in the temporopolar, entorhinal, and perirhinal areas (32). Typically, TLE originates unilaterally from the medial temporal lobe; they may, however, be propagated from other regions which project to limbic areas (33).

Given these potential diffuse structural abnormalities seen in patients with TLE, it could be expected that neuropsychological deficits may not be limited to tasks involving temporal lobe function. Patients with TLE display deficits in memory, general intelligence, language, executive function, and motor speed relative to $\mathrm{HC}(34,35)$. Deficits in verbal memory, language, and psychomotor speed may be affected by factors such as age of onset of epilepsy, general intelligence, the number and dose of antiepileptic medications used, and seizure frequency (35).

The literature describing clinical features, imaging findings, and neuropsychological test profiles is largely in a separate corpus for BD-I and for TLE with only one small study directly comparing the two (12).

The current structured review brings these two bodies of work together in a comparison of the neurocognition literature findings of the two conditions side-by-side. The aim is to determine whether and to what extent the cognitive impairments seen in euthymic BD-I are mirrored by those attributed to a pathology primarily affecting the temporal lobes, that is TLE.

\section{METHOD}

This systematic review was conducted in accordance with the Preferred Reporting Items for Systematic Reviews and MetaAnalyses (PRISMA) guidelines (36). 
Inclusion criteria: (a) controlled comparison, (b) patients diagnosed with BD-I as assessed by a recognized criterion-based diagnostic system, (c) BD-I patients rated as euthymic, defined by their scores on a rating scale $(<8 \mathrm{HamD},<8 \mathrm{YMRS})$; patients with unilateral TLE, from any cause, with diagnosis confirmed in a pre-surgical workup, (d) at least one neuropsychological test, (e) adult participants (18-60 years), (f) in the case of more than one article by the same authors results were not identified as being from the same sample, (f) articles published 1980 or later.

\section{Identification of Studies}

A comprehensive search of the electronic databases PubMed, PsychINFO, and Scopus for peer-reviewed articles published in English was conducted in the last week of May 2016. Search terms were grouped as follows: bipolar, manic depress ${ }^{\star}$, baseline, asympt $^{\star}$, remit ${ }^{\star}$, stable (group 1); or epilep* ${ }^{\star}$ seizure, presurg*, temporal, focal, partial, complex, interictal (group 2) and WAIS, Wechsler, trail making, continuous performance, stroop, digit span, verbal learning, rey, working memory, benton, card sort*, verbal fluency, RAVLT, CAVLT, tower of London (group 3). These search terms were combined as follows: group 1 AND group 3 for BD-I and group 2 AND group 3 for TLE.

\section{Data Extraction}

The abstracts located from the search strategy were entered into EndNoteX7. The PRISMA flowchart (Figure 1) sets out the steps in screening conducted by author EB. After the screening of titles and abstracts the remaining 139 studies were examined in full text, using a purpose built coding sheet to assess whether they met inclusion criteria. This process resulted in the study sample of 56, of which 27 related to BD-I and 29 to TLE.

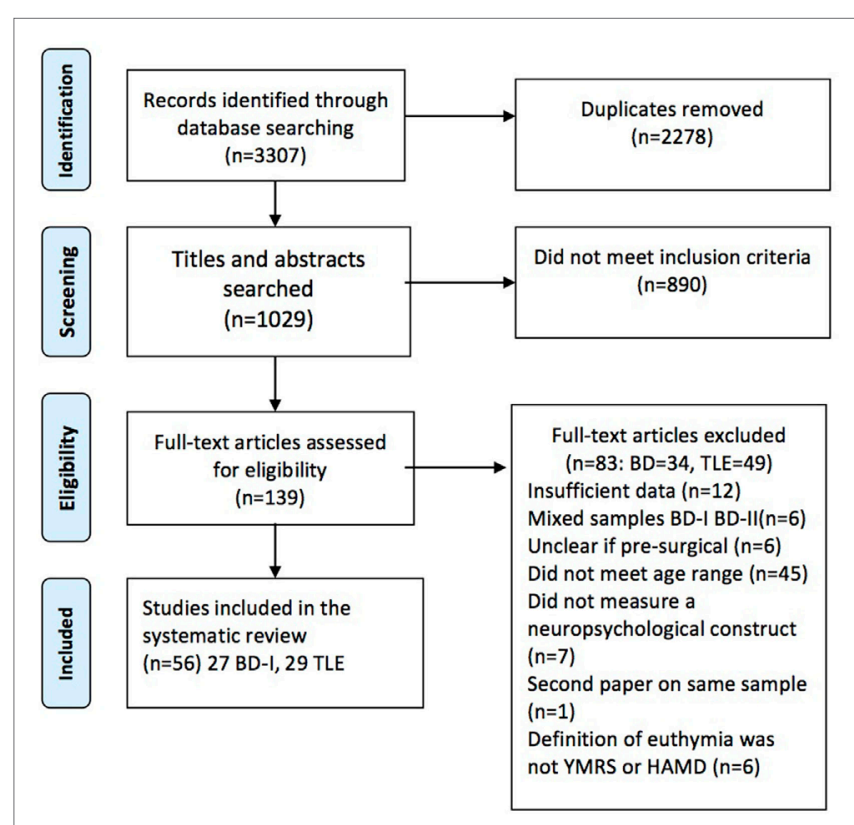

FIGURE 1 | Preferred Reporting Items for Systematic Reviews and Meta-Analyses flow diagram of included studies.

\section{RESULTS}

The search strategy identified an initial 3,307 articles of which 56 met inclusion criteria, 27 related to BD-I and 29 to pre-surgical TLE. Studies of BD-I are summarized in Table $\mathbf{1}$ and of TLE in Table 2 detailing authors, sample sizes, medication (BD-I only as this was not recorded in the TLE samples), neuropsychological test parameters, and summary results.

As demonstrated in Table 1, 25 of 27 studies compared patients with $\mathrm{BD}-\mathrm{I}(n=1,398)$ to $\mathrm{HC}(n=1,142)$. The remaining studies compared manic, euthymic, and depressed groups. The most commonly reported impairments in BD-I were in executive function, attention span, and verbal memory. No studies found enhanced neuropsychological function in euthymic BD-I.

As shown in Table 2, in the epilepsy literature, all of the TLE studies compared neuropsychological test performance pre- to post-surgery. Only 5 of the 29 studies compared pre-surgical TLE $(n=150)$ to HC $(n=111)$; these studies found significant impairments in TLE compared to HC on tests of memory and executive function.

In 26 of the TLE studies, the samples were divided by laterality of seizure focus with the primary pathology affecting the right temporal lobe (RTL $n=846$ ) or the left temporal lobe (LTL $n=1,068)$. In pre-surgical TLE, the direct comparison of LTL and RTL groups indicated that the LTL group showed impaired verbal memory and the RTL group, less consistently, impaired visuospatial memory.

The most common impairment observed in TLE related to surgery was in verbal memory. This finding was also associated with laterality, 24 studies reporting decline in verbal memory from pre- to post-surgery in LTL patients, whereas in RTL there was a less consistent decline in visuospatial memory. No significant differences in attention were found for laterality or pre- to post-surgery in TLE.

Table 3 shows the results of individual neuropsychological tests that were reported in more than one study, for BD-I and TLE. Of note, the total number of neuropsychological tests used across all studies differed between BD-I (27) and TLE (11); this was not evenly distributed across cognitive domains. The number of studies where any executive function instrument was administered in TLE was only 4 ( 2 WCST, 2 COWAT) of 29 compared to 21 of 27 in BD-I. One study compared RTL, LTL and HC and showed that all patients were impaired on the WCST relative to $\mathrm{HC}(87)$. The number of studies where any verbal memory instrument was administered was 26 of 29 in TLE compared to 14 of 27 in BD-I. The comparable figures for visuospatial memory instruments were 25 of 29 in TLE and 11 of 27 in BD-I. Similarly, only 6 studies measured attention in TLE compared to 17 studies in BD-I. Thus, while both fields have seen sustained research activity in identifying neuropsychological deficits, the focus of inquiry in TLE has been on verbal and visuospatial memory and in BD-I executive function.

Notwithstanding the differences in the intensity and focus of neuropsychological testing in both conditions, consistent results emerging from this study emphasize deficits in verbal memory, which have been reported in the majority of studies that have examined this area in both BD-I and TLE. 
TABLE 1 | Summary of included studies on euthymic bipolar disorder.

\begin{tabular}{|c|c|c|c|c|}
\hline Reference & $n$ & $\begin{array}{l}\text { Medications bipolar } \\
\text { sample }\end{array}$ & Neuropsychological test parameters & Results \\
\hline Altshuler et al. (37) & $\begin{array}{l}\text { BD } 40 \\
\text { SZ } 20 \\
\text { HC } 22\end{array}$ & $\begin{array}{l}\mathrm{Li} 25, \mathrm{AC} 12, \mathrm{AD} 4, \mathrm{AP} \\
\text { 6, BZD } 3\end{array}$ & $\begin{array}{l}\text { WAIS-R block design, vocab; TMT, WCST, } \\
\text { Stroop; VFT, CVLT; ROCFT; Star mirror } \\
\text { tracing Task, PR }\end{array}$ & $\begin{array}{l}\text { BD impaired verbal memory and executive } \\
\text { functioning }\end{array}$ \\
\hline Bas et al. (38) & $\begin{array}{l}\mathrm{BD} 60 \\
\mathrm{HC} 41\end{array}$ & $\begin{array}{l}\text { Li 39, AP 37, LTG 9, } \\
\text { VPA } 21\end{array}$ & $\begin{array}{l}\text { Stroop, TMT A and B; WMS-R visual } \\
\text { reproduction; RAVLT }\end{array}$ & BD impaired on RAVLT \\
\hline Bora et al. (39) & $\begin{array}{l}\text { BD } 514 \\
\text { BD-II } 42 \\
\text { HC } 416\end{array}$ & $\begin{array}{l}\text { Li } 63.2 \% \text {, VPA } 47.6 \% \text {, } \\
\text { AP } 47.2 \% \text {, AD } 5.7 \%\end{array}$ & Stroop. WCST & $\begin{array}{l}\text { Deficits exist in subgroups who have severe and } \\
\text { global cognitive deficits }\end{array}$ \\
\hline Chang et al. (40) & $\begin{array}{l}\mathrm{BD} 23 \\
\mathrm{BD}-1123 \\
\mathrm{HC} 23\end{array}$ & $\begin{array}{l}\text { BD Li 13, AP 15, LTG } \\
\text { 7, VPA } 9\end{array}$ & $\begin{array}{l}\text { WAIS-R block design and vocab; CVLT, } \\
\text { VFT }\end{array}$ & All NS \\
\hline Chou et al. (41) & $\begin{array}{l}\mathrm{BD} 23 \\
\mathrm{HC} 33\end{array}$ & AP 13, VPA 19 & $\begin{array}{l}\text { The Color Trails Test, WCST; WMS-III - } \\
\text { Word Lists Test, Face Test; Go/No-GO, } \\
\text { Test for Attentional Performance }\end{array}$ & BD impaired faces memory and WCST \\
\hline Deckersbach et al. (42) & $\begin{array}{l}\mathrm{BD} 25 \\
\mathrm{HC} 25\end{array}$ & Li 11, AC 9, AD 9, AP 3 & ROCFT & $\begin{array}{l}\text { Immediate recall BD impaired, copy and recognition } \\
\text { preserved }\end{array}$ \\
\hline Deckersbach et al. (11) & $\begin{array}{l}\text { BD } 30 \\
\text { Obsessive } \\
\text { compulsive } \\
\text { disorder } 30 \\
\text { HC } 30\end{array}$ & $\begin{array}{l}\text { Li 13, AC 11, AD 8, } \\
\text { AP } 4\end{array}$ & CVLT & CVLT BD impaired learning and delayed free recall \\
\hline Dell’Osso et al. (43) & $\begin{array}{l}\text { BD } 15 \\
\text { BD- } \| 13 \\
\text { HC } 27\end{array}$ & BD Mono 2, Poly 12 & N-Back & NS HC and BD \\
\hline Dittmann et al. (44) & $\begin{array}{l}\text { BD } 65 \\
\text { BD- }-1138 \\
\text { HC } 62\end{array}$ & $\begin{array}{l}\text { BD Li 27, AD 10, AP } \\
\text { 33, CBZ 4, VPA } 28\end{array}$ & $\begin{array}{l}\text { TMT; HAWIE-R; WAIS-III letter number } \\
\text { sequencing; RBANS }\end{array}$ & $\begin{array}{l}\text { Psychomotor speed, working and delayed memory, } \\
\text { verbal learning, executive functioning BD impaired }\end{array}$ \\
\hline Dixon et al. (45) & $\begin{array}{l}\text { BD manic } 15 \text { dep } \\
15 \text {, eu } 15, \text { HC } 30\end{array}$ & $\begin{array}{l}\text { Eu Li 15, AD 8, AP 10, } \\
\text { AC } 2\end{array}$ & $\begin{array}{l}\text { WAIS-R Vocab; Stroop; FAS; Hayling } \\
\text { sentence completion test; Cognitive } \\
\text { Estimates Test }\end{array}$ & $\begin{array}{l}\text { VF total responses, Hayling response initiation } \\
\text { latency, error score and using strategy, stroop EuBD } \\
\text { impaired }\end{array}$ \\
\hline $\begin{array}{l}\text { Doganavşargil-Baysal } \\
\text { et al. (46) }\end{array}$ & $\begin{array}{l}\mathrm{BD} 60 \\
\mathrm{HC} 20\end{array}$ & $\begin{array}{l}\text { Li 11, MS + AP 12, } \\
2 M S 12, \text { VPA } 6\end{array}$ & $\begin{array}{l}\text { WCST, TMT, Stroop; RAVLT; Cancelation } \\
\text { test }\end{array}$ & $\begin{array}{l}\text { Significant differences on all measures between } \mathrm{BD} \\
\text { and } \mathrm{HC}\end{array}$ \\
\hline $\begin{array}{l}\text { Doganavşargil-Baysal } \\
\text { et al. (46) }\end{array}$ & $\begin{array}{l}\text { BD } 54 \\
\text { HC } 18\end{array}$ & $\begin{array}{l}\text { Li 10, MS + AP 27, } \\
2 M S 11, \text { VPA } 5\end{array}$ & WCST; RAVLT & Both measures $\mathrm{BD}<\mathrm{HC}$ \\
\hline Doruk et al. (47) & $\begin{array}{l}\text { Manic } 20 \\
\text { Dep10 } \\
\text { Eu } 21\end{array}$ & Unknown & $\begin{array}{l}\text { Stroop; Serial Digit Learning Test; RAVLT; } \\
\text { Cancelation Test }\end{array}$ & NS HC and EuBD \\
\hline Fistikci et al. (48) & $\begin{array}{l}\mathrm{BD} 25 \\
\mathrm{HC} 25\end{array}$ & Li 25 & WCST; Montreal Cognitive Assessment & $\mathrm{NS} \mathrm{HC}$ and BD \\
\hline Frangou et al. (49) & $\begin{array}{l}\text { BD } 10 \\
\text { Un offspring } 15 \\
\text { HC } 43\end{array}$ & Li 5, AC 3 & WCST; Hayling sentence completion & WCST BD and offspring made more errors \\
\hline Hsiao et al. (50) & $\begin{array}{l}\text { BD } 30 \\
\text { BD- } 1137 \\
\text { HC } 22\end{array}$ & VPA 29 & WAIS-III digit symbol; TMT; WMS-III & $\begin{array}{l}\text { Verbal memory, working memory, psychomotor } \\
\text { speed, executive function BD impaired }\end{array}$ \\
\hline Martino et al. (51) & $\begin{array}{l}\text { BD } 48 \\
\text { BD-II } 37 \\
\text { HC } 34\end{array}$ & $\begin{array}{l}\text { AD 16, AP 28, BZD 23, } \\
\text { MS } 48\end{array}$ & $\begin{array}{l}\text { WAIS vocab, digit span; TMT, WCST, IGT; } \\
\text { Memory battery of Signoret }\end{array}$ & $\begin{array}{l}\text { Verbal memory, attention and executive functions } \\
\text { impaired }\end{array}$ \\
\hline Muralidharan et al. (52) & $\begin{array}{l}\mathrm{BD} 72 \\
\mathrm{HC} 40\end{array}$ & AP 27, Li 34, VPA 38 & $\begin{array}{l}\text { TMT, Stroop; CANTAB; CVLT; WMS-III } \\
\text { LNS }\end{array}$ & $\begin{array}{l}\text { P on VPA more working memory deficits than } \mathrm{Li} \\
\text { or } \mathrm{HC}\end{array}$ \\
\hline Muralidharan et al. (53) & $\begin{array}{l}\mathrm{BD} 68 \\
\mathrm{HC} 38\end{array}$ & $\begin{array}{l}\text { AP 51, Li 32, MS + AP } \\
\text { 50, VPA } 32\end{array}$ & $\begin{array}{l}\text { TMT, Stroop; CANTAB; CVLT; WMS-III } \\
\text { letter number sequencing }\end{array}$ & $\begin{array}{l}\text { Verbal and visuospatial memory, working memory } \\
\text { and executive function BD impaired. }\end{array}$ \\
\hline Normala et al. (54) & $\begin{array}{l}\mathrm{BD} 40 \\
\mathrm{HC} 40\end{array}$ & $\begin{array}{l}\text { AP 6, MS 11, MS + AD } \\
1, M S+A P 22\end{array}$ & WAIS Digit span; TMT; Verbal Fluency & Executive and attention functioning BD impaired \\
\hline
\end{tabular}


TABLE 1 | Continued

\begin{tabular}{|c|c|c|c|c|}
\hline Reference & $n$ & $\begin{array}{l}\text { Medications bipolar } \\
\text { sample }\end{array}$ & Neuropsychological test parameters & Results \\
\hline Pattanayak et al. (55) & $\begin{array}{l}\mathrm{BD} 30 \\
\mathrm{HC} 20\end{array}$ & $\begin{array}{l}\text { AP 5, LTG 2, Li 21, } \\
\text { VPA } 11\end{array}$ & $\begin{array}{l}\text { VIQ; TMT, Stroop; N-Back; Postgraduate } \\
\text { Institute Memory Scale }\end{array}$ & $\begin{array}{l}\text { Attention, information processing speed, executive } \\
\text { function, verbal memory BD impaired }\end{array}$ \\
\hline Radwan (56) & $\begin{array}{l}\mathrm{BD} 30 \\
\mathrm{HC} 30\end{array}$ & Unknown & WAIS; WCST; WMS; CPT & All BD impaired \\
\hline Sepede et al. (57) & $\begin{array}{l}\text { BD } 24 \\
\text { Unaffected rels } \\
33 \\
\text { HC } 24\end{array}$ & $\begin{array}{l}\text { AD 10, AP 15, BZD 8, } \\
\text { Li 3, MS } 9\end{array}$ & СРТ & Sustained attention impaired BD and rels \\
\hline Trivedi et al. (58) & $\begin{array}{l}\text { BD } 15 \\
\text { HC } 15\end{array}$ & CBZ 1, Li 8, VPA 6 & WCST; SWMT; CPT & Executive function BD impaired \\
\hline Trivedi et al. (59) & $\begin{array}{l}\text { BD } 15 \\
\text { SZ } 15, \mathrm{HC} 15\end{array}$ & Unknown & WCST; SWMT; CPT & Executive function BD impaired \\
\hline Yates et al. (60) & $\begin{array}{l}\text { BD dep } 34 \\
\text { BDEu 31, HC } 34\end{array}$ & $\begin{array}{l}\text { AD 9, AP 18, BZD 9, } \\
\text { MS } 29\end{array}$ & WAIS-III & Verbal measure BD impaired \\
\hline Zubieta et al. (61) & $\begin{array}{l}\text { BD } 15 \\
\text { HC } 15\end{array}$ & $\begin{array}{l}\text { AP 3, CBZ 2, Li } 7 \\
\text { VPA } 12\end{array}$ & $\begin{array}{l}\text { WAIS-R: digit span; WCST, Stroop; } \\
\text { WMS-R; verbal fluency; test of variable } \\
\text { attention }\end{array}$ & $\begin{array}{l}\text { Verbal learning, executive function, motor } \\
\text { coordination and sequential memory BD impaired. } \\
\text { NS verbal fluency or attention }\end{array}$ \\
\hline
\end{tabular}

BD, bipolar; BD-II, bipolar II; HC, healthy controls; SZ, Schizophrenia; Li, lithium; AC, anticonvulsant; AD, antidepressant; AP, antipsychotics; BZD, benzodiazepine; CBZ, carbamazepine; LTG, lamotrigine; MS, mood stabilizer; VPA, valproate; Mono, monotherapy; Poly, polytherapy; AMIPB, Adult Memory and Information Processing Battery; BVRT, Benton Visual Retention Test; VLMT, Verbaler Lern- und Merkfähigkeitstest; DCS-R, Diagnosticum für Cerebralschädigung - II; NAART, North American Adult Reading Test; WRAT, Wide Range Achievement Test; WAIS, Wechsler Adult Intelligence Scale; CPT, Continuous Performance Test; CVLT, California Verbal Learning Test; RAVLT, Rey Auditory Verbal Learning Test; WCST, Wisconsin Card Sorting Test.

\section{DISCUSSION}

To our knowledge, this is the first systematic review directly comparing the literature on cognitive function in BD-I and TLE. Consistent with the meta-analyses of cognition in euthymic BD-I, our review showed impairments on a wide range of cognitive measures. In the individual studies reviewed, the most commonly reported impairments in BD-I were in executive function, attention span, and verbal memory. The impairments of executive function in patients with BD-I may be suggestive of an underlying dysfunction in the prefrontal cortex (3).

In TLE studies, a decline in pre- to post-surgery in verbal memory was commonly reported in patients with seizures originating from the LTL, and less consistently, in visuospatial memory in patients with RTL epilepsy. Functional MRI studies have revealed that the right hemisphere is associated with spatial memory (91). In our sample, executive function was not widely examined in patients with epilepsy; however, an impairment was found. For executive function, two instruments were employed across four studies, three of which showed a difference between pre- to post-surgery scores and one study found impaired performance in TLE relative to HC participants.

In keeping with our results, meta-analyses of memory function pre- to post-surgery reported that, following a resection of the LTL, a clear decline in verbal memory is observed, an effect that is particularly salient for immediate verbal recall. However, the pattern of impairment following partial resection of the RTL showed a trend for improvement on tasks of non-verbal memory (92). This suggests that memory impairments are state markers affected by seizures and abnormalities in the temporal lobes. Other factors that affect cognitive performance in TLE are the chronicity of the condition, older age, lower intellect, and greater abnormalities shown on imaging (92). Another meta-analysis found that the evidence regarding post-surgery outcome on visuospatial memory following right anterior temporal lobectomy was less clear (93).

In TLE, it is unclear whether frontal lobe impairments shown on executive function measures are a product of temporal lobe involvement or are a side effect of the propagation of epileptic activity from the epileptic zone (94). Other evidence has suggested that the prefrontal cortex, in particular the orbitofrontal cortex, is influenced by ictal discharges from the mesial temporal lobe (95). Some studies have shown that the temporal neocortex is implicated in executive function implying that a frontotemporal network is used for processing information (96).

This review emphasizes that prior research on cognitive impairments in the fields of BD-I and TLE has employed methodologies that reflect different research questions. The BD-I literature predominantly examines cognition as a characteristic of the disorder itself, on a par with symptoms and potentially amenable to therapeutic intervention. The TLE literature is concerned with the effects of ablative surgery that aims to remove seizure foci but may consequently also directly affect healthy brain tracts. It addresses whether cognitive function improves or declines subsequent to surgery and the moderating effects of laterality.

The majority of BD-I studies compare euthymic patients with $\mathrm{HC}$, whereas in the epilepsy studies, patients act as their own controls in relation to surgical intervention and laterality. In addition, given that the $\mathrm{BD}-\mathrm{I}$ studies are interested in trait markers in the euthymic phase, they routinely report the quantitative differences between patients and $\mathrm{HC}$, rendering the results suitable for incorporation in meta-analytic studies. The TLE literature has focused 
TABLE 2 | Summary of included studies on pre-surgical temporal lobe epilepsy (TLE).

\begin{tabular}{|c|c|c|c|}
\hline Reference & $n$ & Neuropsychological test parameters & Results \\
\hline Baxendale and Thompson (62) & $\begin{array}{l}\text { Right temporal lobe } \\
\text { (RTL) } 133 \\
\text { LTL } 157\end{array}$ & WAIS PIQ, VIQ; AMIPB & $\begin{array}{l}\text { Verbal memory decline post-surg left temporal lobe } \\
(\mathrm{LTL})\end{array}$ \\
\hline Baxendale et al. (63) & $\begin{array}{l}\text { RTL } 146 \\
\text { LTL } 177\end{array}$ & $\begin{array}{l}\text { WAIS PIQ, VIQ; AMIPB; Birt Memory and Information } \\
\text { Processing Battery }\end{array}$ & RTL and LTL at equal risk of post-surg decline \\
\hline Berenbaum et al. (64) & LTL 57 & WAIS Digit Span; CVLT & CVLT decline post-surg \\
\hline Bjørnaes et al. (65) & $\begin{array}{l}\text { RTL } 50 \\
\text { LTL } 41\end{array}$ & $\begin{array}{l}\text { WAIS Digit Span; Benton Visual Retention Test (BVRT); } \\
\text { Design Learning and Retention Test; Verbal List } \\
\text { Learning and Retention; Tactual Performance Test }\end{array}$ & Improvement at 2-year follow up post-surg \\
\hline Chelune et al. (66) & $\begin{array}{l}\text { RTL } 19 \\
\text { LTL } 23\end{array}$ & $\begin{array}{l}\text { WAIS-R VIQ, PIQ; WMS-R, RAVLT; COWAT, Halstead- } \\
\text { Wepman Aphasia Screening Exam, BNT, Speech } \\
\text { Sounds Perception Test; Hooper Visual Organization } \\
\text { Test, Seashore Rhythm Test }\end{array}$ & LTL decline post-surg \\
\hline Chiaravalloti et al. (67) & $\begin{array}{l}\text { RTL } 16 \\
\text { LTL } 10\end{array}$ & $\begin{array}{l}\text { WMS-III Faces Subtest; Graduate Hospital Facial } \\
\text { Memory Test }\end{array}$ & $R T L<L T L$ both pre- and post-surg \\
\hline Chiaravalloti (68) & $\begin{array}{l}\text { RTL } 42 \\
\text { LTL } 28\end{array}$ & CVLT; Graduate Hospital Facial Memory Test & $\begin{array}{l}\text { Verbal memory post-surg decline LTL, RTL improved. } \\
\text { Visuospatial memory post-surg decline RTL, LTL } \\
\text { improvement }\end{array}$ \\
\hline Fernandes et al. (69) & $\begin{array}{l}\text { RTL } 23 \\
\text { LTL } 24 \\
\text { healthy controls } \\
\text { (HC) } 28\end{array}$ & WAIS-R Block design, Vocabulary; WMS-R; RAVLT & $\begin{array}{l}\text { Cognitive scores post-surg decline low pre-surg } \\
\text { scores. Non-verbal memory post-surg RTL decline, } \\
\text { verbal and visuospatial memory LTL decline }\end{array}$ \\
\hline Giovagnoli et al. (70) & $\begin{array}{l}\text { RTL } 12 \\
\text { LTL } 12 \\
\text { HC } 36\end{array}$ & $\begin{array}{l}\text { Raven's Colored Progressive Matrices; Attentive } \\
\text { Matrices; Verbal Selective Reminding Procedure, Story } \\
\text { Recall, Verbal Memory Distractor Test; Visual Selective } \\
\text { Reminding Procedure, ROCFT, Visual Memory } \\
\text { Distractor Test }\end{array}$ & $\begin{array}{l}\text { Verbal memory pre- and post-surgery LTL impaired } \\
\text { relative to } \mathrm{HC} \text {. Visual deficits present in both groups } \\
\text { relative to HC }\end{array}$ \\
\hline Gleissner et al. (71) & $\begin{array}{l}\text { RTL } 63 \\
\text { LTL } 52\end{array}$ & VLMT; DCR-S & Verbal memory LTL decline \\
\hline Glosser et al. (72) & $\begin{array}{l}\text { RTL } 13 \\
\text { LTL } 8 \\
\text { HC } 10\end{array}$ & $\begin{array}{l}\text { Boston Naming Test; CVLT Benton Facial Recognition, } \\
\text { Graduate Hospital Facial Memory }\end{array}$ & $\begin{array}{l}\text { Recognition of familiar faces and learning new faces } \\
\text { RTL impaired. Names of familiar faces LTL impaired }\end{array}$ \\
\hline Helmstaedter et al. (73) & LTL 47 & Verbal Learning and Memory Test; RAVLT & Delayed recall and recognition post-surg improvement \\
\hline Hermann and Wyler (74) & $\begin{array}{l}\text { RTL } 14 \\
\text { LTL } 15\end{array}$ & COWAT; Token Test & Language tests pre-surg LTL deficit \\
\hline Hermann et al. (75) & $\begin{array}{l}\text { RTL } 31 \\
\text { LTL } 26\end{array}$ & CVLT & Verbal memory post-surg RTL increased \\
\hline Hermann et al. (76) & $\begin{array}{l}\text { RTL } 26 \\
\text { LTL } 36\end{array}$ & $\begin{array}{l}\text { WAIS-R Digit Span; Multilingual Aphasia Examination } \\
\text { Visual Naming Test; CVLT }\end{array}$ & CVLT post-surg decline LTL \\
\hline Köylü et al. (77) & $\begin{array}{l}\text { RTL } 12 \\
\text { LTL } 14\end{array}$ & List learning task & LTL post-surg decline \\
\hline Lee et al. (78) & LTL 38 & RAVLT; ROCFT & Memory post-surg decline \\
\hline Loring et al. (79) & $\begin{array}{l}\text { RTL } 13 \\
\text { LTL } 16\end{array}$ & $\begin{array}{l}\text { Selective Reminding Test, Serial Digit Learning, ROCFT, } \\
\text { Form Sequence Learning }\end{array}$ & $\begin{array}{l}\text { Complex figure RTL impaired. Verbal memory decline } \\
\text { LTL }\end{array}$ \\
\hline Malikova et al. (80) & $\begin{array}{l}\text { RTL } 11 \\
\text { LTL } 26\end{array}$ & WAIS-R; WMS-R; Verbal Fluency Test & $\begin{array}{l}\text { FSIQ, global and verbal memory, attention, and } \\
\text { working memory all improved post-surg }\end{array}$ \\
\hline Morino et al. (81) & $\begin{array}{l}\text { RTL } 31 \\
\text { LTL } 31\end{array}$ & WMS-R; Miyake Verbal Retention Test; BVRT & $\begin{array}{l}\text { Memory RTL improved post-surg. Verbal memory LTL } \\
\text { post-surg improved }\end{array}$ \\
\hline Quigg et al. (82) & $\begin{array}{l}\text { RTL } 16 \\
\text { LTL } 14\end{array}$ & TMT; BNT; CVLT; WMS-R Logical Memory Scale & $\begin{array}{l}\text { BNT and CVLT LTL decreased post-surgery. } \\
\text { Language and verbal memory LTL increased. TMT } \\
\text { increased }\end{array}$ \\
\hline Seidenberg et al. (83) & $\begin{array}{l}\text { RTL } 30 \\
\text { LTL } 46\end{array}$ & CVLT & Free recall LTL decline post-surgery \\
\hline
\end{tabular}


TABLE 2 | Continued

\begin{tabular}{|c|c|c|c|}
\hline Reference & $n$ & Neuropsychological test parameters & Results \\
\hline Selwa et al. (84) & $\begin{array}{l}\text { RTL } 14 \\
\text { LTL } 17 \\
\text { Non-surgical TLE } 28\end{array}$ & WAIS-R; WMS & $\begin{array}{l}\text { FSIQ, Logical Memory RTL improved post-surg. } \\
\text { Verbal memory decline LTL post-surg }\end{array}$ \\
\hline Shamim et al. (85) & $\begin{array}{l}\text { RTL } 14 \\
\text { LTL } 16\end{array}$ & WAIS-III; WMS-III & Verbal memory deficit post-surg LTL \\
\hline Stretton et al. (86) & $\begin{array}{l}\text { RTL } 17 \\
\text { LTL } 16 \\
\text { HC } 15\end{array}$ & $\begin{array}{l}\text { WAIS-III Digit Span; Gesture Span Task; Motor } \\
\text { Sequences Task; Dot-Back Paradigm }\end{array}$ & $\begin{array}{l}\text { Working memory pre-surg RTL and LTL worse than } \\
\text { HC. WM improved post-surg LTL }\end{array}$ \\
\hline Tisser et al. (87) & $\begin{array}{l}\text { RTL } 10 \\
\text { LTL } 15 \\
\text { HC } 22\end{array}$ & WAIS-R; WCST & WCST RTL and LTL impaired, improved post-surg \\
\hline Trenerry and Jack (88) & $\begin{array}{l}\text { RTL } 34 \\
\text { LTL } 34\end{array}$ & WAIS-R; WCST & $\begin{array}{l}\text { The WCST is not useful for lateralizing seizure onset } \\
\text { in TLE }\end{array}$ \\
\hline Trenerry et al. (89) & $\begin{array}{l}\text { RTL } 36 \\
\text { LTL } 44\end{array}$ & WAIS-R; WMS-R; RAVLT; Visual Spatial Learning Test & Verbal and visual memory LTL improved post-surg \\
\hline von Rhein et al. (90) & $\begin{array}{l}\text { RTL } 20 \\
\text { LTL } 32\end{array}$ & VLMT; DCS-R; BNT; Token Test & $\begin{array}{l}\text { Verbal Memory impaired post-surgery. Naming decline } \\
\text { post-surgery LTL }\end{array}$ \\
\hline
\end{tabular}

Tests of executive function: WCST, FAS, TMT-B, Stroop, Hayling Sentence Completion Test, CANTAB: Intra Extra Dimensional Set Shifting, CANTAB: Stockings Problem; Tests of Verbal Memory-CVLT, RAVLT, WAIS Vocab, WMS-R: Logical Memory, WMS-R: Verbal Paired Associates, Token Test, VLMT: Verbaler Lern- und Merkfähigkeitstest; Tests of nonverbal memory- ROCFT, WMS: Visual reproduction, WMS-R: Design Memory, WMS-R Visual Reproduction, WMS-III: Face Test, CANTAB: Spatial recognition memory, CANTAB: Pattern Recognition Memory, CANTAB: Paired Associates Learning; Attention span WAIS: Digit Span, Adult Memory and Information Processing Battery (AMIPB); sustained attention CPT; working memory SWMT, N-Back, WAIS: Letter Number Sequencing, WMS-III: Letter Number Sequencing, CANTAB: Spatial Working Memory.

TABLE 3 | Neuropsychological test findings summary table (for tests used more than once) in studies of bipolar disorder (BD) and temporal lobe epilepsy (TLE).

\begin{tabular}{|c|c|c|c|c|c|c|}
\hline \multirow[t]{2}{*}{ Measure } & \multicolumn{2}{|l|}{ BD } & \multicolumn{2}{|l|}{ TLE } & \multirow{2}{*}{$\begin{array}{l}\text { Pre- to } \\
\text { post- } \\
\text { surgical }\end{array}$} & \multirow{2}{*}{$\begin{array}{l}\text { Laterality } \\
\text { effects }\end{array}$} \\
\hline & $\begin{array}{l}\text { Use in studies, number of } \\
\text { participants BD }\end{array}$ & $\begin{array}{l}B D<H C \\
\text { sig }\end{array}$ & $\begin{array}{l}\text { Use in studies, number of } \\
\text { participants TLE }\end{array}$ & sig & & \\
\hline \multicolumn{7}{|l|}{ Executive function } \\
\hline WCST & $\begin{array}{l}12 \text { studies } \\
\mathrm{BD} n=859, \text { healthy controls }(\mathrm{HC}) \\
n=676\end{array}$ & 11 & $\begin{array}{l}2 \text { studies } \\
\text { Right temporal lobe (RTL) } n=44 \text {, left } \\
\text { temporal lobe (LTL) } n=49, \mathrm{HC} n=22\end{array}$ & 1 & $\uparrow 1$ & \\
\hline COWAT(FAS) & $\begin{array}{l}5 \text { studies } \\
\mathrm{BD} n=133, \mathrm{HC} n=130\end{array}$ & 2 & $\begin{array}{l}2 \text { studies } \\
\operatorname{RTL} n=33, \operatorname{LTL} n=38\end{array}$ & 2 & $\uparrow 1$ & $\begin{array}{l}\text { Higher score } \\
\text { LTL group } \\
\text { pre- assoc. } \\
\text { with greater } \\
\text { impairment } \\
\text { post-surg }\end{array}$ \\
\hline TMT-B & $\begin{array}{l}9 \text { studies } \\
\mathrm{BD} n=513, \mathrm{HC}=339\end{array}$ & 7 & & & & \\
\hline Stroop & $\begin{array}{l}10 \text { studies } \\
\mathrm{BD} n=895, \mathrm{HC}=642\end{array}$ & 7 & & & & \\
\hline Hayling Sentence Completion Test & $\begin{array}{l}2 \text { studies } \\
\mathrm{BD} n=25, \mathrm{HC} n=73\end{array}$ & 2 & & & & \\
\hline $\begin{array}{l}\text { CANTAB: Intra Extra Dimensional } \\
\text { Set Shifting }\end{array}$ & $\begin{array}{l}2 \text { studies } \\
\mathrm{BD} n=140, \mathrm{HC} n=78\end{array}$ & 2 & & & & \\
\hline CANTAB: Stockings Problem & $\begin{array}{l}2 \text { studies } \\
\mathrm{BD} n=140, \mathrm{HC} n=78\end{array}$ & 2 & & & & \\
\hline \multicolumn{7}{|l|}{ Verbal memory } \\
\hline CVLT & $\begin{array}{l}5 \text { studies } \\
\mathrm{BD} n=233, \mathrm{HC} n=153\end{array}$ & 3 & $\begin{array}{l}7 \text { studies } \\
\operatorname{RTL} n=158, \text { LTL } n=215, \text { HC } n=10\end{array}$ & 6 & $\downarrow 6$ & $\begin{array}{l}\text { Post-surg LTL } \\
\text { decline } 6 \\
\text { RTL > LTL } 1\end{array}$ \\
\hline
\end{tabular}


TABLE 3 | Continued

Measure

Verbal comprehension: WAIS Vocab

WMS-R: Logical Memory

WMS-R: Verbal Paired Associates

Token Test

VLMT: Verbaler Lern- und Merkfähigkeitstest

\section{Visuospatial memory}

ROCFT

WMS: Visual reproduction

CANTAB: Spatial recognition memory

CANTAB: Pattern Recognition Memory

CANTAB: Paired Associates Learning

WMS-R: Design Memory

WMS-R Visual Reproduction

WMS-III: Face Test

Graduate Hospital Facial Memory

Benton Visual Retention

Diagnosticum für Cerebralschädigung
BD

Use in studies, number of participants BD

3 studies

$\mathrm{BD} n=141, \mathrm{HC}=79$

4 studies

$\mathrm{BD} n=187, \mathrm{HC} n=173$

\begin{tabular}{|c|c|c|c|c|}
\hline $\begin{array}{l}6 \text { studies } \\
\text { RTL } n=206, \text { LTL } n=256, \mathrm{HC} n=28\end{array}$ & 4 & $\uparrow 3$ & $\downarrow 1$ & LTL $<$ RTL 1 \\
\hline $\begin{array}{l}4 \text { studies } \\
\text { RTL } n=119, \operatorname{LTL} n=151, \text { HC } n=28\end{array}$ & 4 & $\uparrow 2$ & $\downarrow 1$ & $\mathrm{LTL}<\mathrm{RTL} 1$ \\
\hline $\begin{array}{l}2 \text { studies } \\
\operatorname{RTL} n=34, \operatorname{LTL} n=47\end{array}$ & 1 & $\uparrow 1$ & & $\begin{array}{l}\text { Post-surg LTL } \\
\text { improved } 1\end{array}$ \\
\hline $\begin{array}{l}2 \text { studies } \\
\text { RTL } n=83, \operatorname{LTL} n=82\end{array}$ & 2 & & $\downarrow 2$ & \\
\hline
\end{tabular}

6 studie

BD $<$ HC Use in studies, number of

TLE

Pre- to Laterality

post- effects

sig

2

participants TLE

5 studies

RTL $n=78, \operatorname{LTL} n=176, \mathrm{HC} n=28$

sig surgical

$5 \quad \downarrow 5$ Post-surg LTL

0 decline 5

0

$$
\mathrm{BD} n=187, \mathrm{HC} n=173
$$

3


TABLE 3 | Continued

\begin{tabular}{|c|c|c|c|c|c|c|}
\hline Measure & \multicolumn{2}{|l|}{ BD } & \multicolumn{2}{|l|}{ TLE } & $\begin{array}{l}\text { Pre- to } \\
\text { post- } \\
\text { surgical }\end{array}$ & $\begin{array}{l}\text { Laterality } \\
\text { effects }\end{array}$ \\
\hline $\begin{array}{l}\text { Adult Memory and Information Processing } \\
\text { Battery (AMIPB) }\end{array}$ & & & $\begin{array}{l}2 \text { studies } \\
\text { RTL } n=279, \operatorname{LTL} n=334\end{array}$ & 2 & $\uparrow 1 \downarrow 1$ & \\
\hline \multicolumn{7}{|l|}{ Sustained attention } \\
\hline \multicolumn{7}{|l|}{ Working memory } \\
\hline SWMT & $\begin{array}{l}2 \text { studies } \\
\mathrm{BD} n=30, \mathrm{HC} n=30\end{array}$ & 0 & & & & \\
\hline N-Back & $\begin{array}{l}2 \text { studies } \\
\mathrm{BD} n=45, \mathrm{HC} n=47\end{array}$ & 0 & & & & \\
\hline CANTAB: Spatial Working Memory & $\begin{array}{l}2 \text { studies } \\
\mathrm{BD} n=140, \mathrm{HC} n=78\end{array}$ & 2 & & & & \\
\hline
\end{tabular}

$\uparrow$ refers to an increase, $\downarrow$ a decrease from pre- to post-surgery, sig refers to the number of studies with a significant result, laterality comments only significant main effects or interactions are discussed.

on statistically significant differences pre- to post-surgery and has not reported information in a form suitable for meta-analysis of pre-surgical cognitive functioning. Therefore, we have employed a systematic review methodology to examine and compare the profile of cognitive deficits in the two conditions. As indicated in the Section "Results," the focus of inquiry of the neuropsychological tests employed in the BD-I studies is predominantly on frontal lobe functions and in TLE on verbal and visuospatial memory.

There are significant differences in the experimental designs examining cognition in the two patient groups. In BD-I studies, cognition is tested broadly with a wide range of measures, with a $\mathrm{HC}$ comparison group and statistical control for medication use. This allows for observed deficits to be interpreted as trait markers of BD-I. This is further supported by familial studies that show similar patterns of impairments among family members and patients (89). By contrast, studies assessing cognitive performance in pre-surgical patients with epilepsy do so generally without HC. Only five studies included HC $(n=111)$, consequently the findings have limited depth compared to those in the BD-I literature $(n=1,142)$. The pre-surgical neuropsychological workup consists mostly of tests of memory function, which is not surprising given that the surgery involves removing parts of or the whole temporal lobe.

In general, the studies on BD-I report the types of medications taken by patients at the time of testing (as shown in Table 1), which may have impacted the results. By contrast, in the epilepsy samples, it was typically not reported whether patients were receiving medication at the time of testing. A study that examined the effects of atypical antipsychotics on cognition in euthymic BD-I patients found that untreated patients showed better performance than those taking medication (93). Many patients with BD-I are treated with anticonvulsants that may worsen or enhance cognition (97). Of the total sample of 884 patients with BD-I included in the systematic review, 299 were taking lithium at the time of testing. A review of the effects of lithium on cognition found that impairments on tasks of psychomotor speed and verbal memory were present, whereas no effect was found on visuospatial ability or attention (97). Thus, cognitive performance may be impaired in various ways by different medications. A recent randomized crossover study examined the effects of methylene blue on cognition and mood-related symptoms in euthymic BD-I and BD-II. Neither low (15 mg) control doses nor high active doses (195 mg) had a significant effect on cognition (98). In rats, methylene blue prevents methylmalonate-induced seizures and oxidative damage in the striatum (99) providing interesting leads for future research into the overlaps between BD and epilepsy.

While this paper has provided an overview of the literature, it is subject to a number of limitations. One such factor is the differences of experimental designs in BD-I and TLE, which meant that a head-to-head meta-analytic comparison was not feasible. As discussed previously, the effect of medication was not uniformly controlled in BD-I and was either not reported or not systematically recorded in the epilepsy samples. In order to determine whether cognitive deficits are related to the illness and not undesirable side effect of medication, examination of otherwise stable drug-free patients would be of interest. The period of time between episodes (mania, depression, or seizures), time of testing, hospitalizations, and the presence of psychotic features were not considered in this review. In BD-I patients, the presence of sub-clinical symptoms is common, even in those patients who are rated as euthymic at the time of testing and may have impacted performance overall (3). 
Although there is wide variation in the diagnostic criteria of euthymia, our study aimed to control for this by using the HAMD and YMRS as cutoffs; however, longitudinal measurements would have been advantageous to characterize proximity to manic or depressive episodes. Residual mood symptoms are also an important consideration in epilepsy where depression is the most common psychiatric comorbidity (18). In community-based samples, the rates of depression in epilepsy range from 20 to $30 \%$ and in hospital samples $20-55 \%(100,101)$. It has been established that depression can cause cognitive impairments, particularly in the domains of attention, psychomotor activity, and memory all of which were relevant to this review (102).

We suggest a strong case may be made for a study comparing neuropsychological tests to assess deficits in BD-I, TLE, and matched HC. In future research, a comprehensive test battery employing tests of attention, executive function, memory, and psychomotor speed, coupled with imaging techniques, should be employed in both disorders relative to HC. This would provide

\section{REFERENCES}

1. Clark L, Goodwin GM. State-and trait-related deficits in sustained attention in bipolar disorder. Eur Arch Psychiatry Clin Neurosci (2004) 254(2):61-8. doi:10.1007/s00406-004-0460-y

2. Malhi GS, Ivanovski B, Szekeres V, Olley A. Bipolar disorder: it's all in your mind? The neuropsychological profile of a biological disorder. Can J Psychiatry (2004) 49(12):813-9. doi:10.1177/070674370404901204

3. Robinson LJ, Thompson JM, Gallagher P, Goswami U, Young AH, Ferrier IN, et al. A meta-analysis of cognitive deficits in euthymic patients with bipolar disorder. J Affect Disord (2006) 93(1-3):105-15. doi:10.1016/j. jad.2006.02.016

4. Torres I, Boudreau V, Yatham L. Neuropsychological functioning in euthymic bipolar disorder: a meta-analysis. Acta Psychiatr Scand Suppl (2007) 116(434):17-26. doi:10.1111/j.1600-0447.2007.01055.x

5. Arts B, Jabben N, Krabbendam L, Van Os J. Meta-analyses of cognitive functioning in euthymic bipolar patients and their first-degree relatives. Psychol Med (2008) 38(06):771-85. doi:10.1017/S0033291707001675

6. Bora E, Yucel M, Pantelis C. Cognitive endophenotypes of bipolar disorder: a meta-analysis of neuropsychological deficits in euthymic patients and their first-degree relatives. J Affect Disord (2009) 113(1):1-20. doi:10.1016/j. jad.2008.06.009

7. Kurtz MM, Gerraty RT. A meta-analytic investigation of neurocognitive deficits in bipolar illness: profile and effects of clinical state. Neuropsychology (2009) 23(5):551. doi:10.1037/a0016277

8. Mann-Wrobel MC, Carreno JT, Dickinson D. Meta-analysis of neuropsychological functioning in euthymic bipolar disorder: an update and investigation of moderator variables. Bipolar Disord (2011) 13(4):334-42. doi:10.1111/j.1399-5618.2011.00935.x

9. Bourne C, Aydemir Ö, Balanzá-Martínez V, Bora E, Brissos S, Cavanagh JTO, et al. Neuropsychological testing of cognitive impairment in euthymic bipolar disorder: an individual patient data meta-analysis. Acta Psychiatr Scand (2013) 128(3):149-62. doi:10.1111/acps.12133

10. Krabbendam L, Arts B, van Os J, Aleman A. Cognitive functioning in patients with schizophrenia and bipolar disorder: a quantitative review. Schizophr Res (2005) 80(2-3):137-49. doi:10.1016/j.schres.2005.08.004

11. Deckersbach T, Savage CR, Reilly-Harrington N, Clark L, Sachs G, Rauch SL. Episodic memory impairment in bipolar disorder and obsessive-compulsive disorder: the role of memory strategies. Bipolar Disord (2004) 6:233-44. doi:10.1111/j.1399-5618.2004.00118.x

12. JonesBP,Duncan CC,Mirsky AF, PostRM, Theodore WH.Neuropsychological profiles in bipolar affective disorder and complex partial seizure disorder. Neuropsychology (1994) 8:55-64. doi:10.1037/0894-4105.8.1.55 valuable information on the effects of both BD-I and TLE on temporal and other cerebral areas as well as the effects of medication on neuropsychological test parameters. This would also be of value in identifying putative temporal lobe involvement in BD-I.

\section{AUTHOR CONTRIBUTIONS}

$\mathrm{EB}, \mathrm{KK}, \mathrm{MG}$, and BT contributed to the design of the project, the analysis and discussion of the results and write up of the paper with $\mathrm{KK}, \mathrm{MG}$, and $\mathrm{BT}$ contributing their specialist perspective. $\mathrm{EB}$ and $\mathrm{KK}$ assessed the suitability of papers for inclusion in the manuscript and contributed to the PRISMA review.

\section{FUNDING}

Author EB's research is supported by an Australian Postgraduate Award and the Goddard Sapin-Jaloustre Trust.

13. Mazza M, Di Nicola M, Della Marca G, Janiri L, Bria P, Mazza S. Bipolar disorder and epilepsy: a bidirectional relation? Neurobiological underpinnings, current hypotheses, and future research directions. Neuroscientist (2007) 13(4):392-404. doi:10.1177/10738584070130041101

14. Mula M, Marotta AE, Monaco F. Epilepsy and bipolar disorders. Expert Rev Neurother (2010) 10(1):13-23. doi:10.1586/ern.09.139

15. Silberman EK, Post R, Nurnberger J, Theodore W, Boulenger J. Transient sensory, cognitive and affective phenomena in affective illness. A comparison with complex partial epilepsy. Br J Psychiatry (1985) 146(1):81-9. doi:10.1192/bjp.146.1.81

16. Kanner AM, Palac S. Depression in epilepsy: a common but often unrecognized comorbid malady. Epilepsy Behav (2000) 1(1):37-51. doi:10.1006/ ebeh.2000.0030

17. Bakken IJ, Revdal E, Nesvåg R, Brenner E, Knudsen GP, Surén P, et al. Substance use disorders and psychotic disorders in epilepsy: a population-based registry study. Epilepsy Res (2014) 108(8):1435-43. doi:10.1016/j. eplepsyres.2014.06.021

18. Lyketsos CG, Stoline AM, Longstreet P, Ranen NG, Lesser R, Fisher R, et al. Mania in temporal lobe epilepsy. Cogn Behav Neurol (1993) 6(1):19-25.

19. Sucksdorff D, Brown AS, Chudal R, Jokiranta-Olkoniemi E, Leivonen S, Suominen A, et al. Parental and comorbid epilepsy in persons with bipolar disorder. J Affect Disord (2015) 188:107-11. doi:10.1016/j.jad.2015. 08.051

20. BostockECS, Kirkby KC, Garry MI, Taylor BVM. Comparison of precipitating factors for mania and partial seizures: indicative of shared pathophysiology? J Affect Disord (2015) 183:57-67. doi:10.1016/j.jad.2015.04.057

21. McDonald C, Zanelli J, Rabe-Hesketh S, Ellison-Wright I, Sham P, Kalidindi S, et al. Meta-analysis of magnetic resonance imaging brain morphometry studies in bipolar disorder. Biol Psychiatry (2004) 56(6):411-7. doi:10.1016/j. biopsych.2004.06.021

22. Brambilla P, Harenski K, Nicoletti M, Sassi RB, Mallinger AG, Frank E, et al. MRI investigation of temporal lobe structures in bipolar patients. J Psychiatr Res (2003) 37(4):287-95. doi:10.1016/S0022-3956(03)00024-4

23. Altshuler LL, Bartzokis G, Grieder T, Curran J, Jimenez T, Leight K, et al. An MRI study of temporal lobe structures in men with bipolar disorder or schizophrenia. Biol Psychiatry (2000) 48(2):147-62. doi:10.1016/ S0006-3223(00)00836-2

24. Altshuler LL, Conrad A, Hauser P, Ximing L, Guze BH, Denikoff K, et al. Reduction of temporal lobe volume in bipolar disorder: a preliminary report of magnetic resonance imaging. Arch Gen Psychiatry (1991) 48(5):482-3. doi:10.1001/archpsyc.1991.01810290094018

25. Johnstone EC, Owens D, Crow TJ, Frith C, Alexandropolis K, Bydder G, et al. Temporal lobe structure as determined by nuclear magnetic resonance in 
schizophrenia and bipolar affective disorder. J Neurol Neurosurg Psychiatry (1989) 52(6):736-41. doi:10.1136/jnnp.52.6.736

26. Swayze VW, Andreasen NC, Alliger RJ, Yuh WT, Ehrhardt JC. Subcortical and temporal structures in affective disorder and schizophrenia: a magnetic resonance imaging study. Biol Psychiatry (1992) 31(3):221-40. doi:10.1016/0006-3223(92)90046-3

27. Harvey I, Persaud R, Ron MA, Baker G, Murray R. Volumetric MRI measurements in bipolars compared with schizophrenics and healthy controls. Psychol Med (1994) 24(03):689-99. doi:10.1017/S0033291700027847

28. Hauser P, Matochik J, Altshuler LL, Denicoff KD, Conrad A, Li X, et al. MRIbased measurements of temporal lobe and ventricular structures in patients with bipolar I and bipolar II disorders. J Affect Disord (2000) 60(1):25-32. doi:10.1016/S0165-0327(99)00154-8

29. Bernasconi N, Andermann F, Arnold DL, Bernasconi A. Entorhinal cortex MRI assessment in temporal, extratemporal, and idiopathic generalized epilepsy. Epilepsia (2003) 44(8):1070-4. doi:10.1046/j.1528-1157.2003.64802.x

30. Natsume J, Bernasconi N, Andermann F, Bernasconi A. MRI volumetry of the thalamus in temporal, extratemporal, and idiopathic generalized epilepsy. Neurology (2003) 60(8):1296-300. doi:10.1212/01.WNL.0000058764. 34968.C2

31. Kuzniecky R, Bilir E, Gilliam F, Faught E, Martin R, Hugg J. Quantitative MRI in temporal lobe epilepsy evidence for fornix atrophy. Neurology (1999) 53(3):496-496. doi:10.1212/WNL.53.3.496

32. Bernasconi N, Duchesne S, Janke A, Lerch J, Collins D, Bernasconi A. Whole-brain voxel-based statistical analysis of gray matter and white matter in temporal lobe epilepsy. Neuroimage (2004) 23(2):717-23. doi:10.1016/j. neuroimage.2004.06.015

33. Travers RF. Limbic epilepsy. J R Soc Med (1991) 84(8):454.

34. Oyegbile TO, Dow C, Jones J, Bell B, Rutecki P, Sheth R, et al. The nature and course of neuropsychological morbidity in chronic temporal lobe epilepsy. Neurology (2004) 62:1736-42. doi:10.1212/01. WNL.0000125186.04867.34

35. Wang W, Liou H, Chen C, Chiu M, Chen T, Cheng T, et al. Neuropsychological performance and seizure-related risk factors in patients with temporal lobe epilepsy: a retrospective cross-sectional study. Epilepsy Behav (2011) 22:728-34. doi:10.1016/j.yebeh.2011.08.038

36. Moher D, Liberati A, Tetzlaff J, Altman DG. Preferred reporting items for systematic reviews and meta-analyses: the PRISMA statement. Ann Intern Med (2009) 151(4):264-9. doi:10.7326/0003-4819-151-4-200908180-00135

37. Altshuler LL, Ventura J, van Gorp WG, Green MF, Theberge DC, Mintz J. Neurocognitive function in clinically stable men with bipolar I disorder or schizophrenia and normal control subjects. Biol Psychiatry (2004) 56(8):560-9. doi:10.1016/j.biopsych.2004.08.002

38. Bas TO, Poyraz CA, Bas A, Poyraz BC, Tosun M. The impact of cognitive impairment, neurological soft signs and subdepressive symptoms on functional outcome in bipolar disorder. J Affect Disord (2015) 174:336-41. doi:10.1016/j.jad.2014.12.026

39. Bora E, Hıdıroğlu C, Özerdem A, Kaçar ÖF, Sarısoy G, Arslan FC, et al. Executive dysfunction and cognitive subgroups in a large sample of euthymic patients with bipolar disorder. Eur Neuropsychopharmacol (2016) 26(8):1338-47. doi:10.1016/j.euroneuro.2016.04.002

40. Chang JS, Choi S, Ha K, Ha TH, Cho HS, Choi JE, et al. Differential pattern of semantic memory organization between bipolar I and II disorders. Prog Neuropsychopharmacol Biol Psychiatry (2011) 35(4):1053-8. doi:10.1016/j. pnpbp.2011.02.020

41. Chou YH, Wang SJ, Lirng JF, Lin CL, Yang KC, Chen CK, et al. Impaired cognition in bipolar I disorder: the roles of the serotonin transporter and brain-derived neurotrophic factor. J Affect Disord (2012) 143(3):131-7. doi:10.1016/j.jad.2012.05.043

42. Deckersbach T, McMurrich S, Ogutha J, Savage CR, Sachs G, Rauch SL. Characteristics of non-verbal memory impairment in bipolar disorder: the role of encoding strategies. Psychol Med (2004) 34(5):823-32. doi:10.1017/ S0033291703001685

43. Dell'Osso B, Cinnante C, Di Giorgio A, Cremaschi L, Palazzo MC, Cristoffanini $M$, et al. Altered prefrontal cortex activity during working memory task in bipolar disorder: a functional magnetic resonance imaging study in euthymic bipolar I and II patients. J Affect Disord (2015) 184:116-22. doi:10.1016/j.jad.2015.05.026

44. Dittmann S, Henning-Fast K, Gerber S, Seemüller F, Riedel M, Severus WE, et al. Cognitive functioning in euthymic bipolar I and bipolar II patients. Bipolar Disord (2008) 10(8):877-87. doi:10.1111/j.1399-5618.2008.00640.x

45. Dixon T, Kravariti E, Frith C, Murray RM, McGuire PK. Effect of symptoms on executive function in bipolar illness. Psychol Med (2004) 34(5):811-21. doi:10.1017/S0033291703001570

46. Doganavşargil-Baysal GO, Zehra G, Akbaş H, Cinemre B, Metin O, Karaman T. Association of serum homocysteine and methionine levels with cognition and functioning in bipolar disorder. Turk Psikiyatri Derg (2013) 24(1):6-14.

47. Doruk A, Yazihan NT, Balikci A, Erdem M, Bolu A, Ates MA. Cognitive functions in bipolar manic, depressed and remission episodes. Klinik Psikofarmakoloji Bulteni (2014) 24(1):59-68. doi:10.5455/ bcp. 20130506122642

48. Fistikci N, Canturk G, Saatcioglu O, Erten E, Keyvan A, Turan N. Executive functions and thyroid volumes in bipolar patients on lithium treatment. Afr J Psychiatry (South Africa) (2014) 17(6):145. doi:10.4172/Psychiatry.1000145

49. Frangou S, Haldane M, Roddy D, Kumari V. Evidence for deficit in tasks of ventral, but not dorsal, prefrontal executive function as an endophenotypic marker for bipolar disorder. Biol Psychiatry (2005) 58(10):838-9. doi:10.1016/j.biopsych.2005.05.020

50. Hsiao Y-L, Wu Y-S, Wu JY-W, Hsu M-H, Chen H-C, Lee S-Y, et al. Neuropsychological functions in patients with bipolar I and bipolar II disorder. Bipolar Disord (2009) 11(5):547-54. doi:10.1111/j.1399-5618.2009.00723.x

51. Martino DJ, Strejilevich SA, Torralva T, Manes F. Decision making in euthymic bipolar I and bipolar II disorders. Psychol Med (2011) 41(6):1319-27. doi:10.1017/S0033291710001832

52. Muralidharan K, Kozicky JM, Bücker J, Silveira LE, Torres IJ, Yatham LN. Are cognitive deficits similar in remitted early bipolar I disorder patients treated with lithium or valproate? Data from the STOP-EM study. Eur Neuropsychopharmacol (2015) 25(2):223-30. doi:10.1016/j. euroneuro.2014.09.005

53. Muralidharan K, Torres IJ, Silveira LE, Kozicky JM, Bücker J, Fernando N, et al. Impact of depressive episodes on cognitive deficits in early bipolar disorder: data from the systematic treatment optimization programme for early mania (STOP-EM). Br J Psychiatry (2014) 205(1):36-43. doi:10.1192/ bjp.bp. 113.135525

54. Normala I, Abdul Hamid AR, Azlin B, Nik Ruzyanei NJ, Hazli Z, Shah SA. Executive function and attention span in euthymic patients with bipolar 1 disorder. Med J Malaysia (2010) 65(3):192-6.

55. Pattanayak RD, Sagar R, Mehta M. Neuropsychological performance in euthymic Indian patients with bipolar disorder type I: correlation between quality of life and global functioning. Psychiatry Clin Neurosci (2012) 66(7):553-63. doi:10.1111/j.1440-1819.2012.02400.x

56. Radwan DN. Cognitive impairment in Egyptian euthymic patients with bipolar I disorder compared with controls. Middle East Curr Psychiatry (2013) 20(4):197-204. doi:10.1097/01.XME.0000433325.69290.c9

57. Sepede G, De Berardis D, Campanella D, Perrucci MG, Ferretti A, Serroni N, et al. Impaired sustained attention in euthymic bipolar disorder patients and non-affected relatives: an fMRI study. Bipolar Disord (2012) 14(7):764-79. doi:10.1111/bdi.12007

58. Trivedi JK, Dhyani M, Sharma S, Sinha PK, Singh AP, Tandon R. Cognitive functions in euthymic state of bipolar disorder: an Indian study. Cogn Neuropsychiatry (2008) 13(2):135-47. doi:10.1080/13546800801897346

59. Trivedi JK, Goel D, Sharma S, Singh AP, Sinha PK, Tandon R. Cognitive functions in stable schizophrenia and euthymic state of bipolar disorder. Ind J Med Res (2007) 126(5):433-9.

60. Yates DB, Dittmann S, Kapczinski F, Trentini CM. Cognitive abilities and clinical variables in bipolar I depressed and euthymic patients and controls. J Psychiatr Res (2011) 45(4):495-504. doi:10.1016/j.jpsychires.2010.09.006

61. Zubieta J-K, Huguelet P, O'Neil RL, Giordani BJ. Cognitive function in euthymic bipolar I disorder. Psychiatry Res (2001) 102(1):9-20. doi:10.1016/ S0165-1781(01)00242-6

62. Baxendale S, Thompson P. Defining meaningful postoperative change in epilepsy surgery patients: measuring the unmeasurable? Epilepsy Behav (2005) 6(2):207-11. doi:10.1016/j.yebeh.2004.12.009 
63. Baxendale S, Thompson PJ, Sander JW. Neuropsychological outcomes in epilepsy surgery patients with unilateral hippocampal sclerosis and good preoperative memory function. Epilepsia (2013) 54(9):131-4. doi:10.1111/ epi. 12319

64. Berenbaum SA, Baxter L, Seidenberg M, Hermann B. Role of the hippocampus in sex differences in verbal memory: memory outcome following left anterior temporal lobectomy. Neuropsychology (1997) 11(4):585-91. doi:10.1037/0894-4105.11.4.585

65. Bjørnæs H, Stabell KE, Røste GK, Bakke SJ. Changes in verbal and nonverbal memory following anterior temporal lobe surgery for refractory seizures: effects of sex and laterality. Epilepsy Behav (2005) 6(1):71-84. doi:10.1016/j. yebeh.2004.10.011

66. Chelune GJ, Naugle RI, Lüders H, Awad IA. Prediction of cognitive change as a function of preoperative ability status among temporal lobectomy patients seen at 6-month follow-up. Neurology (1991) 41(3):399-404. doi:10.1212/ WNL.41.3.399

67. Chiaravalloti ND, Tulsky DS, Glosser G. Validation of the WMS-III facial memory subtest with the graduate hospital facial memory test in a sample of right and left anterior temporal lobectomy patients. J Clin Exp Neuropsychol (2004) 26(4):484-97. doi:10.1080/13803390490496623

68. Chiaravalloti ND, Glosser G. Material-specific memory changes following anterior temporal lobectomy as predicted by the intracarotid amobarbital test. Epilepsia (2001) 42(7):902-11. doi:10.1046/j.1528-1157.2001.02500.x

69. Fernandes DA, Yasuda CL, Lopes TM, Enrico G, Alessio A, Tedeschi H, et al. Long-term postoperative atrophy of contralateral hippocampus and cognitive function in unilateral refractory MTLE with unilateral hippocampal sclerosis. Epilepsy Behav (2014) 36:108-14. doi:10.1016/j.yebeh.2014.04.028

70. Giovagnoli AR, Casazza M, Ciceri E, Avanzini G, Broggi G. Preserved memory in temporal lobe epilepsy patients after surgery for low-grade tumour. A pilot study. Neurol Sci (2007) 28(5):251-8. doi:10.1007/s10072-007-0831-z

71. Gleissner U, Helmstaedter C, Schramm J, Elger CE. Memory outcome after selective amygdalohippocampectomy in patients with temporal lobe epilepsy: one-year follow-up. Epilepsia (2004) 45(8):960-2. doi:10.1111/j.0013-9580.2004.42203.x

72. Glosser G, Salvucci AE, Chiaravalloti ND. Naming and recognizing famous faces in temporal lobe epilepsy. Neurology (2003) 61(1):81-6. doi:10.1212/01. WNL.0000073621.18013.E1

73. Helmstaedter C, Grunwald T, Lehnertz K, Gleissner U, Elger CE. Differential involvement of left temporolateral and temporomesial structures in verbal declarative learning and memory: evidence from temporal lobe epilepsy. Brain Cogn (1997) 35(1):110-31. doi:10.1006/brcg.1997.0930

74. Hermann BP, Wyler AR. Effects of anterior temporal lobectomy on language function: a controlled study. Ann Neurol (1988) 23(6):585-8. doi:10.1002/ ana. 410230610

75. Hermann BP, Wyler AR, Bush AJ, Tabatabai FR. Differential effects of left and right anterior temporal lobectomy on verbal learning and memory performance. Epilepsia (1992) 33(2):289-97. doi:10.1111/j.1528-1157.1992. tb02318.x

76. Hermann BP, Wyler AR, Somes G, Dohan FC Jr, Berry AD III, Clement L. Declarative memory following anterior temporal lobectomy in humans. Behav Neurosci (1994) 108(1):3-10. doi:10.1037/0735-7044.108.1.3

77. Köylü B, Walser G, Ischebeck A, Ortler M, Benke T. Functional imaging of semantic memory predicts postoperative episodic memory functions in chronic temporal lobe epilepsy. Brain Res (2008) 1223:73-81. doi:10.1016/j. brainres.2008.05.075

78. Lee T, Mackenzie RA, Walker AJ, Matheson JM, Sachdev P. Effects of left temporal lobectomy and amygdalohippocampectomy on memory. J Clin Neurosci (1997) 4(3):314-9. doi:10.1016/S0967-5868(97)90098-9

79. Loring DW, Lee GP, Martin RC, Meador KJ. Material-specific learning in patients with partial complex seizures of temporal lobe origin: convergent validation of memory constructs. J Epilepsy (1988) 1(2):53-9. doi:10.1016/ s0896-6974(88)80057-4

80. Malikova H, Kramska L, Vojtech Z, Lukavsky J, Liscak R. Stereotactic radiofrequency amygdalohippocampectomy: two years of good neuropsychological outcomes. Epilepsy Res (2013) 106(3):423-32. doi:10.1016/j. eplepsyres.2013.07.009
81. Morino M, Ichinose T, Uda T, Kondo K, Ohfuji S, Ohata K. Memory outcome following transsylvian selective amygdalohippocampectomy in 62 patients with hippocampal sclerosis. J Neurosurg (2009) 110(6):1164-9. doi:10.3171/2008.9.jns08247

82. Quigg M, Broshek DK, Barbaro NM, Ward MM, Laxer KD, Yan G, et al. Neuropsychological outcomes after gamma knife radiosurgery for mesial temporal lobe epilepsy: a prospective multicenter study. Epilepsia (2011) 52(5):909-16. doi:10.1111/j.1528-1167.2011.02987.x

83. Seidenberg M, Hermann BP, Dohan FC Jr, Wyler AR, Perrine A, Schoenfeld J. Hippocampal sclerosis and verbal encoding ability following anterior temporal lobectomy. Neuropsychologia (1996) 34(7):699-708. doi:10.1016/0028-3932(95)00146-8

84. Selwa LM, Berent S, Giordani B, Henry TR, Buchtel HA, Ross DA. Serial cognitive testing in temporal lobe epilepsy: longitudinal changes with medical and surgical therapies. Epilepsia (1994) 35(4):743-9. doi:10.1111/ j.1528-1157.1994.tb02505.x

85. Shamim S, Wiggs E, Heiss J, Sato S, Liew C, Solomon J, et al. Temporal lobectomy: resection volume, neuropsychological effects, and seizure outcome. Epilepsy Behav (2009) 16(2):311-4. doi:10.1016/j.yebeh.2009.07.040

86. Stretton J, Sidhu MK, Winston GP, Bartlett P, McEvoy AW, Symms MR, et al. Working memory network plasticity after anterior temporal lobe resection: a longitudinal functional magnetic resonance imaging study. Brain (2014) 137(5):1439-53. doi:10.1093/brain/awu061

87. Tisser L, Palmini A, Paglioli E, Portuguez M, Azambuja N, da Costa JC, et al. Pre and post-operative Wisconsin card sorting test performance in patients with temporal lobe epilepsy due to hippocampal sclerosis. Dement Neuropsychol (2007) 1(2):173-80. doi:10.1590/s1980$57642008 \mathrm{dn} 10200010$

88. Trenerry MR, Jack CR. Wisconsin card sorting test performance before and after temporal lobectomy. J Epilepsy (1994) 7(4):313-7. doi:10.1016/0896-6974(94)90062-0

89. Trenerry MR, Jack CR Jr, Ivnik RJ, Sharbrough FW, Cascino GD, Hirschorn KA, et al. MRI hippocampal volumes and memory function before and after temporal lobectomy. Neurology (1993) 43(9):1800-5. doi:10.1212/ WNL.43.9.1800

90. von Rhein B, Nelles M, Urbach H, Von Lehe M, Schramm J, Helmstaedter C. Neuropsychological outcome after selective amygdalohippocampectomy: subtemporal versus transsylvian approach. J Neurol Neurosurg Psychiatry (2012) 83(9):887-93. doi:10.1136/jnnp-2011-302025

91. d'Esposito M, Aguirre G, Zarahn E, Ballard D, Shin R, Lease J. Functional MRI studies of spatial and nonspatial working memory. Brain Res Cogn Brain Res (1998) 7(1):1-13. doi:10.1016/S0926-6410(98)00004-4

92. Lee T, Yip JT, Jones-Gotman M. Memory deficits after resection from left or right anterior temporal lobe in humans: a meta-analytic review. Epilepsia (2002) 43(3):283-91. doi:10.1046/j.1528-1157.2002.09901.x

93. Vaz SAM. Nonverbal memory functioning following right anterior temporal lobectomy: a meta-analytic review. Seizure (2004) 13(7):446-52. doi:10.1016/j.seizure.2003.12.004

94. Devinsky O. The myth of silent cortex and the morbidity of epileptogenic tissue: implications for temporal lobectomy. Epilepsy Behav (2005) 7(3):383-9. doi:10.1016/j.yebeh.2005.07.020

95. Lieb JP, Dasheiff RM, Engel J. Role of the frontal lobes in the propagation of mesial temporal lobe seizures. Epilepsia (1991) 32(6):822-37. doi:10.1111/ j.1528-1157.1991.tb05539.x

96. Rusnáková Š, Daniel P, Chládek J, Jurák P, Rektor I. The executive functions in frontal and temporal lobes: a flanker task intracerebral recording study. J Clin Neurophysiol (2011) 28(1):30-5. doi:10.1097/WNP.0b013e31820512d4

97. Pachet AK, Wisniewski AM. The effects of lithium on cognition: an updated review. Psychopharmacology (2003) 170(3):225-34. doi:10.1007/ s00213-003-1592-x

98. Alda M, McKinnon M, Blagdon R, Garnham J, MacLellan S, O'donovan C, et al. Methylene blue treatment for residual symptoms of bipolar disorder: randomised crossover study. Br J Psychiatry (2016) 210(1):54-60. doi:10.1192/bjp.bp.115.173930

99. Furian AF, Fighera MR, Oliveira MS, de Oliveira Ferreira AP, Fiorenza NG, de Carvalho Myskiw J, et al. Methylene blue prevents methylmalonate-induced 
seizures and oxidative damage in rat striatum. Neurochem Int (2007) 50(1):164-71. doi:10.1016/j.neuint.2006.07.012

100. Blum D, Reed M, Metz A. Prevalence of major affective disorders and manic/hypomanic symptoms in persons with epilepsy: a community survey. Neurology (2002) 58(7):175.

101. Kanner AM, Balabanov A. Depression and epilepsy how closely related are they? Neurology (2002) 58(8 Suppl 5):S27-39. doi:10.1212/WNL.58.8_ suppl_5.S27

102. Gallassi R, Morreale A, Pagni P. The relationship between depression and cognition. Arch Gerontol Geriatr (2001) 33:163-71. doi:10.1016/ S0167-4943(01)00136-4
Conflict of Interest Statement: The authors declare that the research was conducted in the absence of any commercial or financial relationships that could be construed as a potential conflict of interest.

Copyright (C) 2017 Bostock, Kirkby, Garry and Taylor. This is an open-access article distributed under the terms of the Creative Commons Attribution License (CC BY). The use, distribution or reproduction in other forums is permitted, provided the original author(s) or licensor are credited and that the original publication in this journal is cited, in accordance with accepted academic practice. No use, distribution or reproduction is permitted which does not comply with these terms. 